\title{
Other and III-Defined Sites within Respiratory System and Intrathoracic Organs ICD-O-3
}

National Cancer Institute

\section{Source}

National Cancer Institute. Other and III-Defined Sites within Respiratory System and

Intrathoracic Organs ICD-O-3. NCI Thesaurus. Code C148338.

Anatomic sites within the respiratory system and intrathoracic organs that are

referenced in topographic category C39 of ICD-O-3. 